\title{
Epithelioid sarcoma associated with neurofibromatosis type I
}

\author{
Sung Oh Hwang, \\ Soo Hyang Lee, \\ Han Byul Lee \\ Department of Plastic and \\ Reconstructive Surgery, Inje University \\ Ilsan Paik Hospital, Inje University \\ College of Medicine, Goyang, Korea
}

\begin{abstract}
In general, patients with neurofibromatosis type I have a higher risk than those with other types of neurofibromatosis of developing soft-tissue sarcomas related to the nervous system. We here present a 42-year-old man with neurofibromatosis type I who developed a protruding mass over only 2 weeks. The histopathological diagnosis was epithelioid sarcoma. Epithelioid sarcomas are rare and, to the best of our knowledge, no epithelioid sarcomas have been reported in patients with neurofibromatosis type I. Radical excision of the primary lesion was performed and postoperative radiotherapy and chemotherapy administered, as is recommended for epithelioid sarcoma. Our case emphasizes that patients with neurofibromatosis type I may develop malignant tumors.
\end{abstract}

Keywords: Epithelioid sarcoma / Neurofibromatosis type I / Sarcomas

\section{INTRODUCTION}

Neurofibromatosis type I (NF I) or von Recklinghausen disease, is an autosomal dominant disorder caused by inactivating mutations in the germline of the NF I gene. Characteristic features include pigmented skin lesions (freckling, cafe-au-lait spots), Lisch nodules, malignant or benign tumors, and organ or tissue damage (visceral, skeletal, and neural). Large complete gene deletions are associated with a high risk of development of malignant tumors of the nervous system, such as schwannomas, gliomas, and malignant peripheral nerve sheath tumors (MPNSTs). In particular, from $8 \%$ to $13 \%$ of patients with NF I develop MPNSTs [1-3]. However, NF I fibromatosis is generally benign and transformation into malignancy is rare. Also, malignant tumors unrelated to the nervous system rarely develop in patients with neurofibromatosis. However, few cases of NF I associated with sarcoma have been reported, including pleo-

\footnotetext{
Correspondence: Soo Hyang Lee

Department of Plastic and Reconstructive Surgery, Inje University Ilsan Paik Hospital, Inje University College of Medicine, 170 Juhwa-ro, Ilsanseo-gu, Goyang 10380, Korea

E-mail: shyanglee@naver.com

Received November 4, 2019 / Revised December 13, 2019 / Accepted December 16, 2019
}

morphic sarcoma [4], rhabdomyosarcoma [5], leiomyosarcoma [6], and Ewing sarcoma [7]. Here, we describe an unusual case of epithelioid sarcoma in a patient with NF I.

\section{CASE REPORT}

A 42-year-old man with a history of NF I presented with a growing mass on his posterior neck that he had first noted about 2 weeks earlier. Numerous hyperpigmented skin macules were observed on his back, abdomen and chest, and multiple cafe-au-lait spots on his shoulders and abdomen. Several neurofibromas were identified on his neck and abdomen (Fig. 1). He reported that his father and uncle had neurofibromas and cafe-au-lait spots on their skin.

The initial examination showed a $3.5 \times 3.5 \mathrm{~cm}$ protruding mass with ulcerative bleeding on the posterior neck (Fig. 2). Malignancy was suspected because of the lesion's rapid growth and surface ulceration. Because of the time required for biopsy, the patient refused to undergo biopsy first and instead requested immediate resection of the tumor. Accordingly, the mass was excised under general anesthesia and frozen sections examined. Excision was performed to the supramuscular layer 

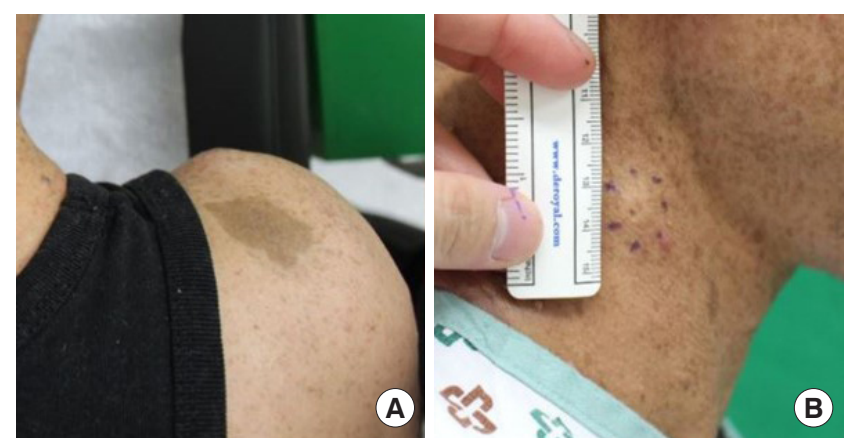

Fig. 1. Photographs showing cafe-au-lait spots on the patient's right shoulder (A) and several neurofibromas on his neck (B).

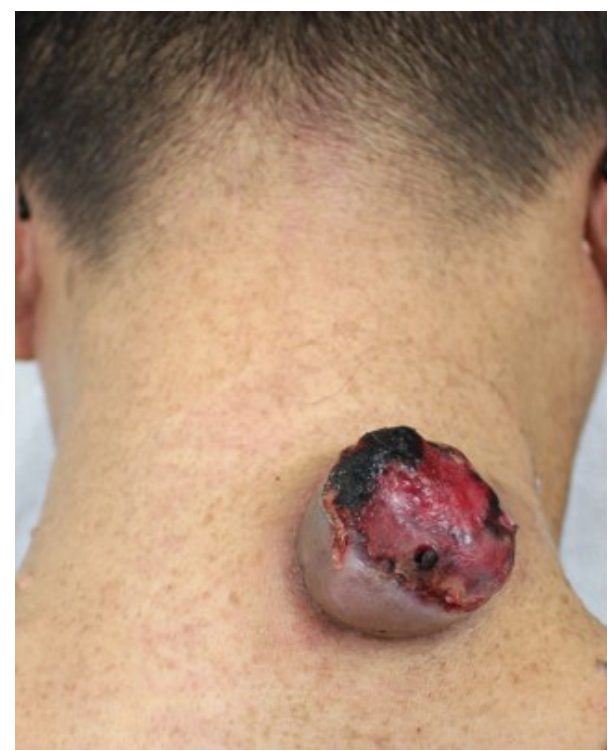

Fig. 2. Photograph showing an approximately $3.5 \times 3.5 \mathrm{~cm}$ protruding ulcerated mass with bleeding on the patient's posterior neck.

with a $2 \mathrm{~cm}$ margin. Intraoperative examination of frozen sections resulted in a diagnosis of high-grade sarcoma with epithelioid features. After confirming that the resection margin was clear, the defect was covered by primary closure (Fig. 3). Pathological examination of the excised lesion confirmed the frozen section diagnosis of high-grade epithelioid sarcoma; the resection margin was clear. Immunohistochemistry studies showed that the tumor cells were strongly positive for cytokeratin (Fig. 4), but negative for CD34 and S100. They also showed weak nuclear expression of integrase interactor 1. Postoperatively, the patient underwent positron emission tomography-computed tomography (PET-CT) and enhanced neck CT; subcutaneous nodular lesions with enhancement were detected. Abnormal uptake of fluorodeoxyglucose was also detected in the lateral neck region bilaterally (Fig. 5). We therefore planned more extensive excision and sentinel lymph node biopsy with radical neck dissection and postoperative radiotherapy and chemo-

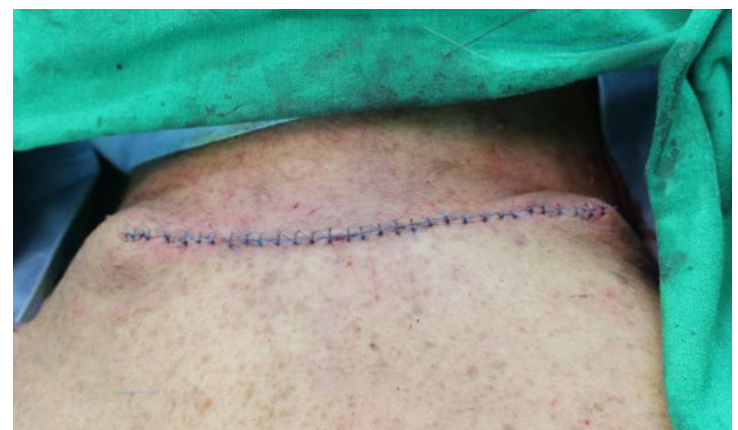

Fig. 3. Postoperative photograph.

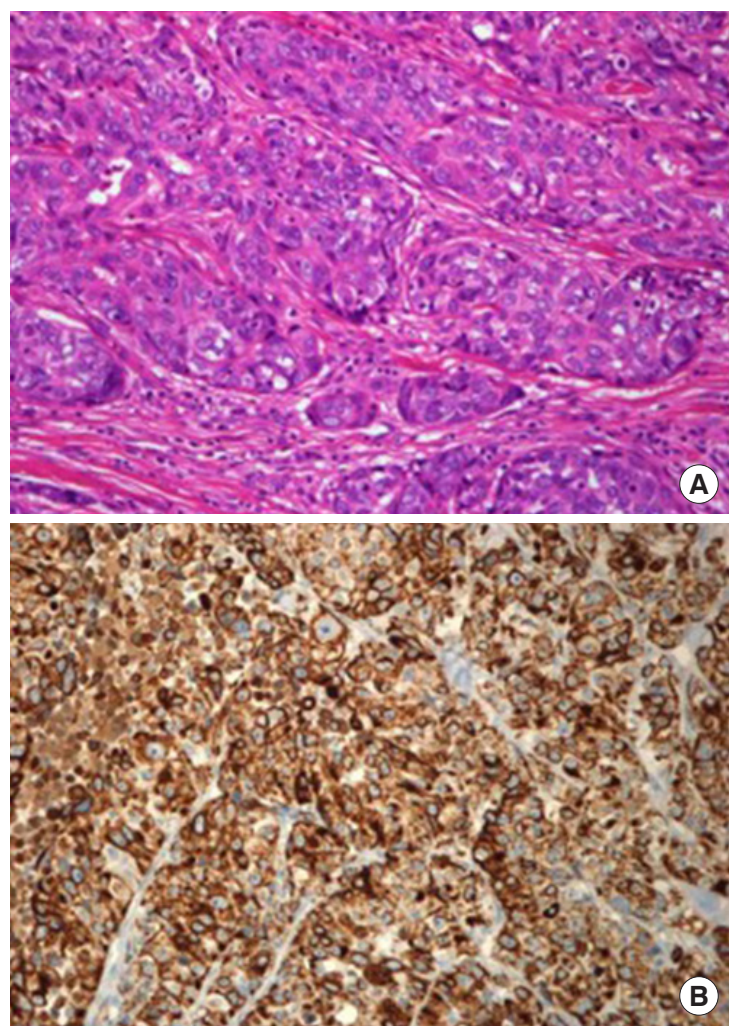

Fig. 4. (A) Photomicrograph of the operative specimen showing nests of tumor cells with epithelioid features in the dermis (H\&E, $\times 200$ ). (B) Immunohistochemistry study showing that the tumor is strongly positive for cytokeratin $(\times 200)$.

therapy. However, the patient consented only to excision of the suspicious lesions detected on radiologic evaluation. During the second operation on both lateral neck regions, abnormal tissue was found in the subdermal layer and excised with a clear resection margin. The excised lesions were diagnosed as epithelioid sarcomas. The patient agreed to postoperative therapy to minimize the risk of tumor metastasis. During a 6-month follow-up, he underwent radiotherapy with chemotherapy (doxorubicin and ifosfamide). No recurrence has been detected to date. 


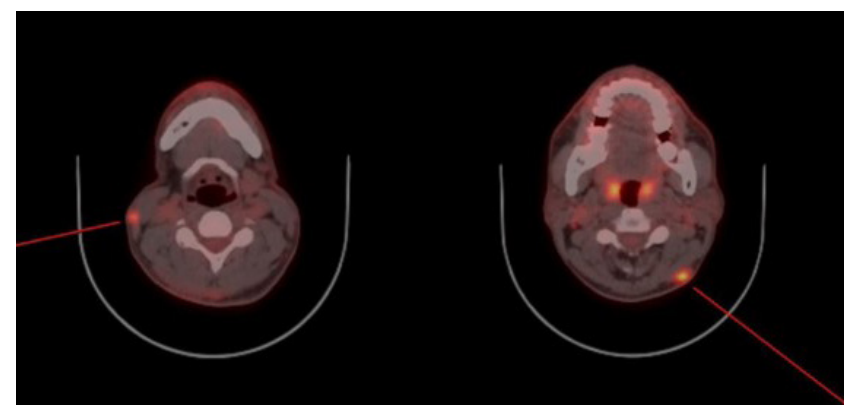

Fig. 5. Positron emission tomography-computed tomography images showing fluorodeoxyglucose uptake in subcutaneous lesions.

\section{DISCUSSION}

NF I, an autosomal dominant disorder, is characterized by the development of multiple peripheral nerve sheath tumors called neurofibromas. There are seven diagnostic criteria for NF I according to the National Institutes of Health (Table 1) [8], two or more of which must be met to make a diagnosis of NF I. Neurofibromas and cafe-au-lait spots are benign and generally do not need to be treated. However, plexiform neurofibromas have the potential for malignant development. There is an $8 \%$ to $13 \%$ risk of developing malignant nerve sheath tumors. These malignant tumors are treated with extensive local resection; additionally, imatinib has been shown to reduce the size of plexiform neurofibromas [9]. Monitoring for neurologic changes is important because such changes may indicate tumor development. Although neurofibromas are benign, it has been well established that patients with NF I have a higher than general risk of developing soft-tissue sarcomas related to the nervous system $[1,10-12]$. Optic glioma is the most common central nervous system tumor associated with NF I [13], whereas malignant nerve sheath tumors are the most common peripheral nervous system tumor associated with NF I [14]. In some cases, NF I malignant transformation is associated with heterologous differentiation of components [4], commonly including angiosarcoma, chondrosarcoma, rhabdomyosarcoma, and osteosarcoma.

Epithelioid sarcoma, first described by Laskowski in 1961 [15], is a rare soft-tissue tumor with a propensity for local recurrence, regional lymph node involvement, and distant metastasis. It commonly presents as a slow-growing, firm-to-hard palpable mass with surface ulceration and invasive borders and disseminates via subdermal lymphatic vessels [16]. After the establishment of histopathological diagnosis, epithelioid sarcomas are treated by radical excision. Sentinel lymph node biopsy with therapeutic lymph node dissection is indicated in patients suspected of having regional lymph node or systemic metasta-
Table 1. Diagnostic criteria for neurofibromatosis type I

1. Six or more cafe-au-lait spots, greater than $5 \mathrm{~mm}$ prepubertal and greater than $15 \mathrm{~mm}$ post-pubertal

2. Two or more neurofibromas or one or more plexiform neurofibroma

3. Axillary or groin freckling

4. Optic glioma

5. Two or more Lisch nodules

6. Sphenoid dysplasia, dysplasia or thinning of long bone cortex

7. First-degree relative with neurofibromatosis type I

Two or more should be met for the diagnosis of neurofibromatosis type I (National Institutes of Health) [8].

ses. Radiation can also be considered and adjuvant chemotherapy is indicated when metastatic disease is suspected or confirmed [17-20].

There is currently no known direct association between neurofibromatosis and epithelioid sarcomas. To the best of our knowledge, this is the first report of an epithelioid sarcoma developing in a patient with NF I. We did not establish a direct association between the above two diseases in our patient. However, considering that patients with neurofibromatosis are at high risk of developing multiple malignancies, it would be of interest to compare our patient's disease course with that of patients with NF I and the more commonly associated malignant tumors. The present case highlights that physicians should be aware of the possibility of malignant tumors in patients with NF I, as well as their histological characteristics and prognoses.

\section{NOTES}

\section{Conflict of interest}

No potential conflict of interest relevant to this article was reported.

\section{Ethical approval}

The study was performed in accordance with the principles of the Declaration of Helsinki. Written informed consent was obtained.

\section{Patient consent}

The patient provided written informed consent for the publication and the use of his images.

\section{ORCID}

Sung Oh Hwang https://orcid.org/0000-0001-5537-9124

Soo Hyang Lee https://orcid.org/0000-0002-1679-5624

Han Byul Lee https://orcid.org/0000-0002-1147-7678 


\section{REFERENCES}

1. Ward BA, Gutmann DH. Neurofibromatosis 1: from lab bench to clinic. Pediatr Neurol 2005;32:221-8.

2. Sorensen SA, Mulvihill JJ, Nielsen A. Long-term follow-up of von Recklinghausen neurofibromatosis: survival and malignant neoplasms. N Engl J Med 1986;314:1010-5.

3. Evans DG, Baser ME, McGaughran J, Sharif S, Howard E, Moran A. Malignant peripheral nerve sheath tumours in neurofibromatosis 1. J Med Genet 2002;39:311-4.

4. de Oliveira MG, Pozatti Moure S, Sergio Batista P, Moraes Chaves AC, Rados PV, Sant'Ana Filho M. NF1 diagnosis criteria and associated sarcomatous tumor review of the literature and case report. Oral Maxillofac Surg 2008;12:231-5.

5. Ferrari A, Bisogno G, Macaluso A, Casanova M, D’Angelo P, Pierani $\mathrm{P}$, et al. Soft-tissue sarcomas in children and adolescents with neurofibromatosis type 1. Cancer 2007;109:1406-12.

6. Kim A, Stewart DR, Reilly KM, Viskochil D, Miettinen MM, Widemann BC. Malignant peripheral nerve sheath tumors state of the science: leveraging clinical and biological insights into effective therapies. Sarcoma 2017;2017:7429697.

7. Chowdhry M, Hughes C, Grimer RJ, Sumathi V, Wilson S, Jeys L. Bone sarcomas arising in patients with neurofibromatosis type 1. J Bone Joint Surg Br 2009;91:1223-6.

8. Stumpf DA. Neurofibromatosis. Conference statement: National Institutes of Health Consensus Development Conference. Arch Neurol 1988;45:575-8.

9. Ferner RE, Huson SM, Thomas N, Moss C, Willshaw H, Evans DG, et al. Guidelines for the diagnosis and management of individuals with neurofibromatosis 1. J Med Genet 2007;44:81-8.

10. Friedman JM. Neurofibromatosis 1: clinical manifestations and diagnostic criteria. J Child Neurol 2002;17:548-54.
11. Hatori M, Hosaka M, Watanabe M, Moriya T, Sasano H, Kokubun S. Osteosarcoma in a patient with neurofibromatosis type 1: a case report and review of the literature. Tohoku J Exp Med 2006;208:343-8.

12. Shearer P, Parham D, Kovnar E, Kun L, Rao B, Lobe T, et al. Neurofibromatosis type I and malignancy: review of 32 pediatric cases treated at a single institution. Med Pediatr Oncol 1994;22:78-83.

13. Lewis RA, Gerson LP, Axelson KA, Riccardi VM, Whitford RP. von Recklinghausen neurofibromatosis. II. Incidence of optic gliomata. Ophthalmology 1984;91:929-35.

14. Razek AAKA. MR imaging of neoplastic and non-neoplastic lesions of the brain and spine in neurofibromatosis type I. Neurol Sci 2018;39:821-7.

15. Laskowski J. Sarcoma aponeuroticum. Nowotory 1961;11:617.

16. Enzinger FM. Epitheloid sarcoma: a sarcoma simulating a granuloma or a carcinoma. Cancer 1970;26:1029-41.

17. Spillane AJ, Thomas JM, Fisher C. Epithelioid sarcoma: the clinicopathological complexities of this rare soft tissue sarcoma. Ann Surg Oncol 2000;7:218-25.

18. Callister MD, Ballo MT, Pisters PW, Patel SR, Feig BW, Pollock $\mathrm{RE}$, et al. Epithelioid sarcoma: results of conservative surgery and radiotherapy. Int J Radiat Oncol Biol Phys 2001;51:384-91.

19. Prat J, Woodruff JM, Marcove RC. Epithelioid sarcoma: an analysis of 22 cases indicating the prognostic significance of vascular invasion and regional lymph node metastasis. Cancer 1978;41:1472-87.

20. Ross HM, Lewis JJ, Woodruff JM, Brennan MF. Epithelioid sarcoma: clinical behavior and prognostic factors of survival. Ann Surg Oncol 1997;4:491-5. 